\title{
Evaluation of Virtual Noncontrast Images Obtained from Dual- Energy CTA for Diagnosing Subarachnoid Hemorrhage
}

\author{
X.Y. Jiang, S.H. Zhang, Q.Z. Xie, Z.J. Yin, Q.Y. Liu, M.D. Zhao, X.L. Li, and X.J. Mao
}

\begin{abstract}
BACKGROUND AND PURPOSE: The virtual noncontrast images generated with iodine subtraction from dual-energy CTA images are expected to replace the true noncontrast images for radiation-dose reduction. This study assessed the feasibility of virtual noncontrast images for diagnosing SAH.
\end{abstract}

MATERIALS AND METHODS: Eighty-four patients with or without SAH underwent true noncontrast brain CT (the criterion standard for diagnosing SAH). Among them, 37 patients underwent an additional head dual-energy angiography, and the other patients underwent head and neck dual-energy angiography. Virtual noncontrast images were produced on a dedicated dual-energy postprocessing workstation and reconstructed in orientation and section width identical to those in true noncontrast images. The findings on the virtual noncontrast and true noncontrast images were compared at both the individual level and the lesion level. Image noise of the virtual noncontrast and true noncontrast images was also measured and compared. The volume CT dose index and dose-length product were recorded for the radiation-dose analysis.

RESULTS: The sensitivity, specificity, positive predictive value, and negative predictive value of virtual noncontrast images at the individual level and the lesion level were $94.5 \%, 100 \%, 100 \%, 90.6 \%$ and $86.7 \%, 96.9 \%, 91.8 \%, 94.8 \%$, respectively. The agreement in the diagnosis of SAH on true noncontrast and virtual noncontrast images reached $92.3 \%$ at the individual level and $85.1 \%$ at the lesion level. The virtual noncontrast images showed a higher image noise level. The volume CT dose index and dose-length product were obviously reduced without the true noncontrast brain $\mathrm{CT}$ scan.

CONCLUSIONS: Virtual noncontrast images are a reliable tool for diagnosing $\mathrm{SAH}$, with the advantage of reducing the radiation dose.

ABBREVIATIONS: TNC $=$ true noncontrast; $\mathrm{VNC}=$ virtual noncontrast

S

ubarachnoid hemorrhage is defined as the extravasation of blood into the spaces filled with CSF in the central nervous system. ${ }^{1}$ The characteristic appearance of extravasated blood on CT is hyperattenuated, which is markedly different from the CT attenuation of brain tissue, CSF, and bone. ${ }^{1,2}$ Therefore noncontrast CT is the criterion standard for diagnosing SAH. Rupture of an intracranial aneurysm is the major cause of SAH and accounts for approximately $80 \%$ of nontraumatic SAH cases. ${ }^{3}$ CTA is a fast, noninvasive, and readily available method to screen for the presence of an aneurysm. ${ }^{4}$ As a result, patients with suspected SAH

Received October 2, 2014; accepted after revision November 9.

From the Departments of Radiology (X.Y.J., Z.J.Y., Q.Y.L., M.D.Z., X.L.L., X.J.M.) and Pediatrics (Q.Z.X.), Affiliated Hospital of Binzhou Medical University, Shandong, P.R. China; and Department of Radiology (S.H.Z.), Shandong Cancer Hospital and Institute, Shandong, P.R. China.

Please address correspondence to Xing Yue Jiang, MD, Department of Radiology, Affiliated Hospital of Binzhou Medical University, 661 Second Huanghe Rd, Binzhou, Shandong 256603, P.R. China; e-mail: xyjiang188@sina.com

http://dx.doi.org/10.3174/ajnr.A4223 should undergo a noncontrast CT scan of the brain for the diagnosis of SAH, followed by CTA to look for the underlying cause of the SAH. Both examinations are considered necessary. A recent article comparing diagnostic strategies for patients in the emergency department with suspected SAH showed that CT along with CTA was considered the most sensitive and rapid test for diagnosing aneurysm, but had the highest radiation exposure. ${ }^{5}$ However, the limitation of a large amount of radiation exposure to patients might be overcome by the advent of dual-energy. Dual-energy simultaneously acquires datasets at 2 different photon spectra in a single acquisition. ${ }^{6}$ Images postprocessed with 3-material decomposition can be used to differentiate iodine from other materials, ${ }^{7}$ and virtual noncontrast (VNC) images can be created by removing the iodine-containing voxels of dual-energy CTA images. If VNC images can replace true noncontrast (TNC) images, the radiation dose can potentially be reduced. To our knowledge, few studies have compared VNC and TNC images in SAH. ${ }^{8}$ The purpose of our study was to assess the utility of VNC images obtained 
from dual-energy CTA for diagnosing SAH compared with TNC images.

\section{MATERIALS AND METHODS Patients}

Between May 2012 and January 2013, 55 patients with SAH and 29 patients who were suspected of having head disease but were diagnosed as not having SAH were included in the study. The group with SAH (case group) included 29 men and 26 women with an age range of $23-79$ years; mean age, $57 \pm 12$ years; median age, 58 years. The non-SAH group (control group) included 22 men and 7 women with an age range of 14-76 years; mean age, $51 \pm 12$ years; median age, 50 years. All patients underwent TNC brain CT with either additional head dual-energy CTA $\left(n_{\mathrm{SAH}}=\right.$ $\left.27, n_{\text {non-SAH }}=10\right)$ or head and neck dual-energy CTA $\left(n_{\mathrm{SAH}}=\right.$ $\left.28, n_{\text {non-SAH }}=19\right)$ with available datasets.

In the case group $(n=55), 25$ patients had SAH only and the rest had other intracranial changes in addition to $\mathrm{SAH}$ as follows: cerebral hemorrhage $(n=15)$, lacunar infarction or cerebral infarction $(n=8)$, lacunar infarction and cerebral hemorrhage $(n=$ $2)$, epidural hematoma $(n=1)$, cerebral hemorrhage and subdural hematomas $(n=1)$, arachnoid cyst $(n=1)$, hydrocephalus $(n=1)$, and subdural collection of fluid $(n=1)$. The causes for SAH included aneurysm (33 cases were diagnosed or suspected by CTA and 26 were confirmed by surgery or DSA), definite history of head injury $(n=6)$, vascular malformation $(n=1)$, and unclear causes $(n=15)$. The interval between the onset of SAH and the CT examination was in the range of 4-391 hours, with a mean of $57.57 \pm 89.43$ hours and a median of 19.50 hours; $67.27 \%$ of patients had a time interval of $<24$ hours, and $72.73 \%$ of patients had a time interval of $<48$ hours.

In the control group $(n=29)$, there were 9 patients with essentially normal findings, 17 with lacunar infarction, 1 with subdural collection of fluid, 1 with a history of a cerebral hemorrhage operation 16 months previously, and 1 with a vascular malformation of the cerebellar hemisphere (but without SAH). The CTA findings in the control group included the following: normal findings $(n=4)$, atherosclerosis $(n=19)$, suspicious aneurysm $(n=$ 5 , without confirmation by an operation or DSA), and vascular malformation $(n=1)$.

This study was approved by our institutional review board, and all patients or their relatives provided written informed consent before the CT examination.

\section{CT Data Acquisition and Postprocessing}

All examinations were performed by using a second-generation dual-source CT scanner (Somatom Definition Flash; Siemens, Erlangen, Germany). TNC scans were obtained in a single-energy mode, and CTA was performed in a dual-energy mode.

The TNC brain CT was performed in all patients by using an axial scan technique with a collimation of $128 \times 0.6 \mathrm{~mm}$ and a tube voltage of $120 \mathrm{kV}$. The current flow-dose modulation (CARE Dose4D; Siemens) was enabled with a range of 256-436 mAs.

For CTA scans, we used 2 different tube voltages and currents simultaneously: tube A at $140 \mathrm{kV}$ and tube B at $100 \mathrm{kV}$. Dose modulation (CARE Dose4D, Siemens) was enabled with a range of 110-140 and 107-157 mAs for tube A and tube B, respectively; a rotation time of 0.28 seconds per rotation; $128 \times 0.6 \mathrm{~mm}$ collimation with a z-flying focal spot; and a pitch of 0.9. An automated power injector (Stellant D; MedRad, Indianola, Pennsylvania) was used to administer $60 \mathrm{~mL}$ of contrast material (ioversol, $320 \mathrm{~g}$ $\mathrm{I} / 100 \mathrm{~mL}$ ) at a rate of $4 \mathrm{~mL} / \mathrm{s}$ and followed by $20 \mathrm{~mL}$ of saline solution into the antecubital vein via an 18-ga catheter. The CT scan was triggered by a bolus-tracking technique with the ROI placed in the common carotid artery at the level of C4 and the image acquisition started 4 seconds after the attenuation reached the predefined threshold (100 Hounsfield units). Scan direction was cranial-caudal. The examination region of head dual-energy CTA and head and neck dual-energy CTA was from the cranial end of the skull to the inferior margin of the mandible and from the cranial end of the skull to the arcus aortae, respectively.

TNC images were reconstructed with a 5-mm thickness/interval in the main console and were transferred to the PACS. For each dual-energy CTA examination, we reconstructed 2 image datasets with one at $140 \mathrm{kV}$ and another at $100 \mathrm{kV}$ with a $0.75-\mathrm{mm}$ thickness/0.5-mm interval and transferred them to the workstation (syngo MultiModality; Siemens) for further processing. Images were loaded onto a dedicated dual-energy postprocessing workstation (syngo MultiModality Workplace, Version 2010A; Siemens). The VNC images were produced with a dual-energyanalysis material-decomposition software (syngo Dual Energy Brain Hemorrhage; Siemens) and were reconstructed with orientation and section width identical to those of the TNC images. The findings on VNC images were compared with those on the TNC images.

\section{CT Image Analysis}

Diagnosis of SAH. Two experienced radiologists (X.Y.J., X.J.M., with 19 and 12 years' experience in neuroradiology, respectively) who were blinded to the patient clinical data were asked to analyze the performance of the TNC and VNC images in consensus, respectively (including case group and control group; note the cases were mixed and labeled by patient identification). There was an interval of 2 months between the evaluation of TNC and VNC images. The evaluations were recorded in a table. We divided the subarachnoid space into 27 sections, including symmetric framework (cistern of the pontocerebellar angle, pontine cistern, ambience cistern, tentorium of the cerebellum, suprasellar cistern, cistern of the lateral fossa of the cerebrum, and lateral ventricle), asymmetric framework (cistern of the cerebral longitudinal fissure, third ventricle, fourth ventricle), and cortical sulci (in units of lobes: frontal lobe, parietal lobe, occipital lobe, temporal lobe, and insula). For the symmetric framework and lobe, each side was counted as an individual section. The TNC images were used as the criterion standard for diagnosing $\mathrm{SAH}$, and the sensitivity, specificity, positive predictive value, negative predictive value, and $\kappa$ value were calculated accordingly. Statistical analyses were performed at the individual (patient) and lesion levels (each bleeding section representing a lesion).

Objective Evaluation of Image Quality. For objective evaluation of image quality, image noise ${ }^{9}$ of the TNC images and VNC images was measured as the SD of a $1-\mathrm{cm}^{2}$ ROI placed in homogeneous and normal regions of white matter on the left 
centrum ovale. For the same patient, the ROI would be placed in the same region of the same section on the TNC and VNC images. For all measurements, the size and shape of the ROIs were kept constant.

Subjective Evaluation of Image Quality. The image quality was rated by a radiologist (M.D.Z, with 27 years' experience in neuroradiology) blinded to the patient clinical data by using a 3-point scale: 1 = excellent, when the VNC images were comparable with TNC images; 2 = sufficient, when VNC images were usable in the detection of SAH; and 3 = insufficient, when the VNC images were insufficient for the detection of SAH.

Radiation Dose. The CT dose index and dose-length product displayed on the CT system were used to calculate the radiation dose.

\section{Statistical Analyses}

The McNemar test was used to compare TNC and VNC images at the individual level, whereas at the lesion level, the Wilcoxon matched-pairs signed rank sum test was used. The Cohen $\kappa$ statistic was used to assess the agreement of the diagnostic results between the TNC and VNC images. The guidelines of Landis and $\mathrm{Koch}^{10}$ were followed to interpret $\kappa$ values, and the scale is listed as the following: $0.00-0.20$ indicated slight agreement; $0.21-0.40$, fair agreement; $0.41-0.60$, moderate agreement; 0.6-0.80, substantial agreement; and 0.80-1.00, almost perfect agreement.

Image noise of the TNC and VNC images was calculated and expressed as mean $\pm \mathrm{SD}$. Results were tested for statistical significance by using the paired $t$ test.

The radiation dose of head dual-energy CTA, head and neck dual-energy CTA, and TNC CT was calculated and expressed as mean $\pm \mathrm{SD}$.

$P$ values $<.05$ were statistically significant. All statistics were performed with a statistical software program (SPSS, Version 16.0; IBM, Armonk, New York).

Table 1: Cases of SAH detected on TNC and VNC images at the

\begin{tabular}{lccc} 
& \multicolumn{2}{c}{ TNC } & \\
\cline { 2 - 3 } \multicolumn{1}{c}{ VNC } & Positive & Negative & Total \\
\hline Positive & 52 & 0 & 52 \\
Negative & 3 & 29 & 32 \\
Total & 55 & 29 & 84 \\
\hline
\end{tabular}
individual level

\section{RESULTS}

\section{Diagnosis of the SAH}

At the Individual Level. The number of cases with SAH diagnosed on TNC and VNC images is presented in Table 1.

As reported in Table 1, VNC images failed to show SAH in 3 patients (false-negative results), whereas no patient was misdiagnosed with SAH. No statistical difference was found in diagnosing SAH between the TNC and VNC images $(P=.250)$. An almost perfect agreement in the diagnosis of SAH on TNC and VNC images was demonstrated $(\kappa$ value $=0.923, P<.001)$.

We studied the 3 patients (who had false-negative results) retrospectively and found that they had a long interval of 8,11 , and 12 days between the onset of SAH and CTA examinations. At the same time, each of the 3 patients had just 1,2, or 4 lesions, respectively. All patients had been confirmed to have SAH by CT in another hospital and then were transferred to our hospital for further evaluation and management when their conditions were stabilized. The increased time interval between the onset of SAH and the CTA examination allowed most of the SAH to be absorbed. The attenuation of the bleeding became very low, and there were only a few remaining lesions, which made it difficult for VNC images to detect these lesions. Compared with TNC images, some of the lesions could be found on VNC images (Fig $1 A,-B)$; but some of the lesions could not be found on VNC images, even after comparing them with those on TNC images retrospectively (Fig $1 C,-D$ ). Of the 3 patients, 1 patient was found to have an aneurysm of the anterior communicating artery and the other 2 patients had negative findings on CTA examinations.

At the Lesion Level. The number of lesions with SAH detected on TNC and VNC images is presented in Table 2.

There were 648 lesions detected on TNC images, and 612 lesions on VNC images. There was no statistical difference in detecting lesions between TNC and VNC images $(P=.121)$. VNC images missed 86 lesions in total (false-negative results) and misdiagnosed 50 sections with SAH in total (false-positive results).

Table 2: Number of SAH lesions detected on TNC and VNC images at the lesion level

\begin{tabular}{|c|c|c|c|}
\hline \multirow[b]{2}{*}{ VNC } & \multicolumn{2}{|c|}{ TNC } & \multirow[b]{2}{*}{ Total } \\
\hline & Positive & Negative & \\
\hline Positive & 562 & 50 & 612 \\
\hline Negative & 86 & 1570 & 1656 \\
\hline Total & 648 & 1620 & 2268 \\
\hline
\end{tabular}

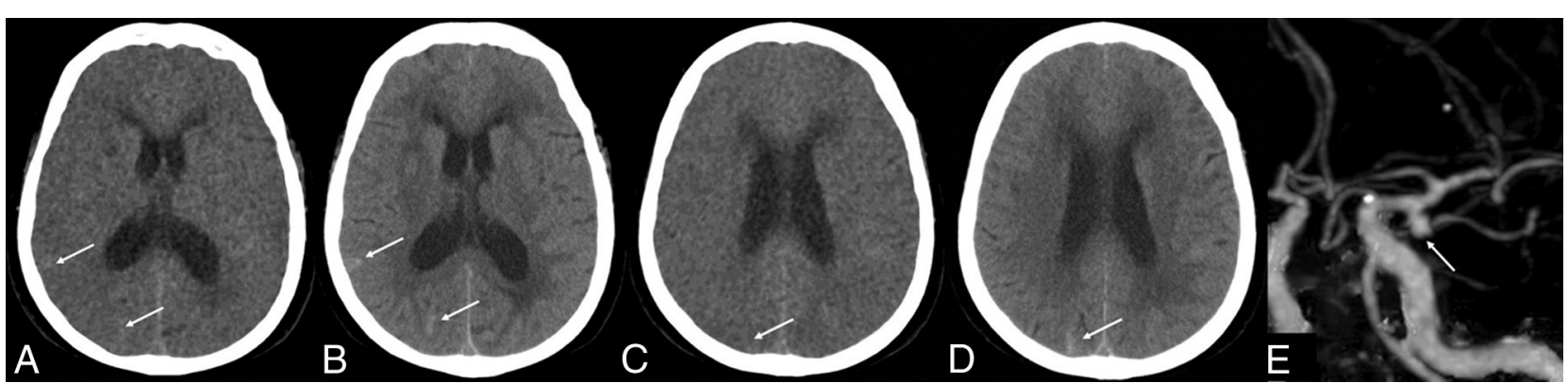

FIG 1. A 71-year-old woman with SAH missed on VNC images. The onset time of SAH was 12 days prior. CTA shows an aneurysm of the left MCA. $A$ and $C$, VNC image; $B$ and $D$, TNC image; $E, C T A$ image. 
The diagnosis of SAH on TNC and VNC images reached an almost perfect agreement of $85.1 \%(\kappa$ value $=0.851, P<.001)$.

We divided the patients (case group) into 3 groups according to the number of lesions: group 1 with $\leq 3$ lesions ( $n=5$ ), group 2 with $\leq 5$ lesions $(n=8)$, and group 3 with $>5$ lesions $(n=42)$. In group 1 , there were 2 patients in whom the diagnosis of $\mathrm{SAH}$ was missed on VNC images; in group 2, there was 1 patient in whom the diagnosis of SAH was missed. No patient was misdiagnosed in group 3.

\section{Evaluation of VNC Images for Diagnosing SAH}

As shown in Table 3, the sensitivity, specificity, positive predictive value, and negative predictive value of VNC images were high at both the individual level and the lesion level, with the individual level higher than the lesion level, except in regard to the negative predictive value.

Objective Evaluation of Image Quality. The VNC images showed a higher image noise level and markedly reduced contrast between gray and white matter. The mean image noise of TNC and VNC images was $2.98 \pm 0.41$ and $3.35 \pm 0.59$, respectively, and a significant difference was observed $(t=-5.286, P<.001)$.

Subjective Evaluation of Image Quality. As for the image quality, 56 sets of VNC images were of excellent quality, 28 were sufficient, and no set was insufficient. The average rating was 1.33 , indicating limited image quality noted on VNC images.

Radiation Dose. The radiation doses of head dual-energy CTA, head and neck dual-energy CTA, and the TNC CT scan are presented in Table 4.

The volume CT dose index and dose-length product were sig-

Table 3: Evaluation of VNC images for diagnosing SAH

\begin{tabular}{lcccc}
\multicolumn{1}{c}{ VNC Images } & $\begin{array}{c}\text { Sensitivity } \\
(\%)\end{array}$ & $\begin{array}{c}\text { Specificity } \\
(\%)\end{array}$ & $\begin{array}{c}\text { Positive } \\
\text { Predictive } \\
\text { Value (\%) }\end{array}$ & $\begin{array}{c}\text { Negative } \\
\text { Predictive } \\
\text { Value (\%) }\end{array}$ \\
\hline At the individual level & 94.5 & 100 & 100 & 90.6 \\
At the lesion level & 86.7 & 96.9 & 91.8 & 94.8 \\
\hline
\end{tabular}

Table 4: Radiation dose

\begin{tabular}{lccc}
\hline & \multicolumn{2}{c}{ Dual-Energy CTA } & \\
\cline { 2 - 3 } & Head & Head and Neck & TNC Brain CT \\
\hline CTDI $_{\text {vol (mGy) }}$ & $9.06 \pm 0.52$ & $10.43 \pm 0.38$ & $55.71 \pm 7.28$ \\
DLP $(\mathrm{mGy} \times \mathrm{cm})$ & $229.11 \pm 41.50$ & $398.44 \pm 21.71$ & $773.49 \pm 104.06$ \\
\hline
\end{tabular}

Note:- $-\mathrm{CTDI}_{\text {vol }}$ indicates volume $\mathrm{CT}$ dose index; DLP, dose-length product.

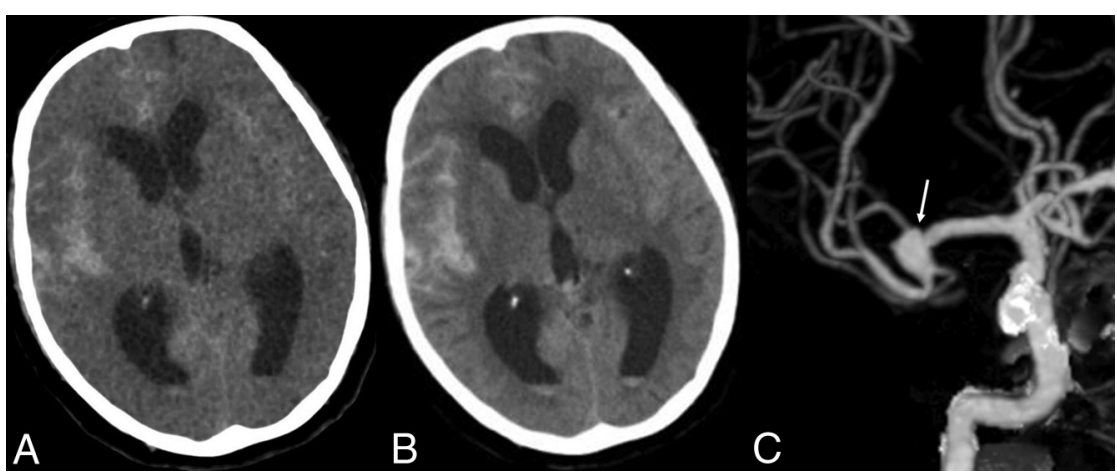

FIG 2. A 78-year-old woman. The onset time of SAH was 17 hours prior. CTA shows an aneurysm in the right MCA. A, VNC image; $B, \mathrm{TNC}$ image; $C, C T A$ image. nificantly lower by using the dual-energy protocol and replacing TNC brain CT with VNC, leading to a radiation-dose reduction of $80.31 \%-89.92 \%$ and $58.98 \%-83.13 \%$, respectively.

\section{DISCUSSION}

The analysis of dual-energy data enables material decomposition, which is the process of separating the materials within each image voxel. ${ }^{11}$ Material decomposition typically entails a selection of 3 materials of interest with the software available with dual-source dual-energy scanners. As such, VNC images can be generated by extracting iodine from other materials. ${ }^{6}$

VNC images had been studied primarily in the abdominal aorta and in patients after endovascular repair. ${ }^{12}$ To date, many studies of the clinical application of VNC images have been performed, mainly in the abdomen for characterizing renal masses, ${ }^{13}$ applications in pancreatic $\mathrm{CT},{ }^{14}$ and detecting urinary or biliary stones. ${ }^{15,16}$ Most of those studies proved that VNC images were useful and feasible, though the imaging quality was inferior to that in the TNC images. Very few studies were performed on the detection of intracranial bleeding. ${ }^{8}$ Our study focused on evaluating the value of VNC images in diagnosing SAH. Compared with the previous studies, the present study had the following advantages: First, only SAH was studied and discussed in this study, while the previous studies discussed $\mathrm{SAH}$, combining intracerebral and subdural bleeding, which was easier to detect on VNC images. Second, the subarachnoid space was divided into 27 sections, enabling the quantification of lesions of SAH. In our study, we found that there was some difficulty in using VNC images to correctly diagnose SAH when the number of lesions was $\leq 3$, because in 2 of 5 patients $(40 \%)$ in group 1 , SAHs were missed. It was easy for VNC images to diagnose $\mathrm{SAH}$ when the number of lesions was $>5$ because all of the patients in group 3 were correctly diagnosed. Third, patients with negative findings were included; therefore, the results were more convincing.

In our study, although VNC images showed a higher image noise level and markedly reduced contrast between gray and white matter compared with the TNC images, VNC images detected $\mathrm{SAH}$ in almost all patients and most of the lesions. There was no significant difference between VNC and TNC images in detecting $\mathrm{SAH}$ at both individual and lesion levels, proving the diagnostic value of VNC images in SAH. The high negative predictive value of $\mathrm{VNC}$ images indicated that the absence of SAH in a patient could be stated confidently, while the higher positive predictive value indicated that the diagnosis of the existence of SAH should be certain. An almost perfect agreement for the diagnosis of SAH between TNC and VNC images was achieved, especially at the individual level. Compared with the TNC images, almost identical locations, extent, and sizes of subarachnoid bleeding were displayed on the VNC images, and only the margin of hemorrhagic focus was not as clear as that seen in the TNC images (Fig 2), probably due to the relatively high noise in VNC images.

In addition, we found that the VNC images showed better conspicuity of 


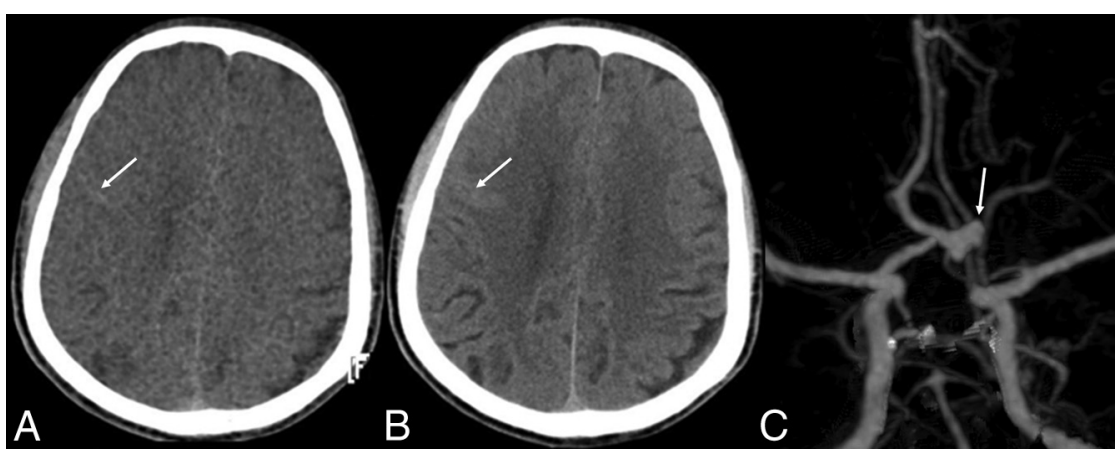

FIG 3. A 58-year-old man. The onset time of SAH was 2.5 days prior. CTA and DSA show an aneurysm of the anterior communicating artery. Both VNC and TNC images find SAH, but the cortical sulci of right frontal lobe appears to have slightly higher attenuation on the VNC images than on TNC images. A, VNC image; $B$, TNC image; $C$, CTA image.

hemorrhagic focus than the TNC images in a few patients, especially on the surface of the brain. This may be explained by the overall markedly reduced contrast between gray and white matter on the VNC images. The attenuation of gray matter was decreased at the same time, so the hemorrhagic focus in the cortical sulci and schizencephaly was more clearly depicted in the VNC images (Fig 3). In a study of assessment of VNC images in patients with gallstones or bile duct stones also found that the VNC images showed better stone conspicuity than the TNC images in some patients. ${ }^{16}$

At the individual level, there was no false-positive diagnosis for $\mathrm{SAH}$, while $\mathrm{SAH}$ in 3 patients was missed on the VNC images. In a retrospective study, we compared the TNC and VNC images of the 3 patients and found that the missed diagnoses were mainly due to the rather small size of bleeding and the relatively low number of bleeding sites on the VNC images. Of course, the higher image noise level could also be one of the reasons. As mentioned above, all 3 patients had a long interval between the onset of SAH and the CTA examination. At the time of CTA, most of the $\mathrm{SAH}$ was absorbed with very low residual attenuation of the bleeding and very few remaining lesions. Therefore when the hemorrhagic focus of SAH was very small with very low attenuation, it was difficult to differentiate a small lesion from artifacts on the VNC images. However, in general, VNC images could satisfy the clinical requirements for diagnosing SAH with high sensitivity and specificity because most patients usually had enough lesions and clear enough signal on VNC images, especially during the acute stage when most SAHs were diagnosed.

At the lesion level, there were 50 false-positive lesions and 86 false-negative lesions. The most frequent sites for false-positive lesions were the occipital lobe (18\%), suprasellar cistern (16\%), tentorium of the cerebellum, and the frontal lobe (both were $12 \%)$, while the frequent false-negative lesions were in the lateral ventricle $(15 \%)$, parietal lobe, and ambient cistern (both accounted for 13\%). The VNC images tended to have decreased attenuation and amount of blood in the lateral ventricle (Fig 2). Although the ambient cistern is a common place for aneurysmal $\mathrm{SAH}$ and also a frequent site for the false-negative lesions, in our study, there were no patients with SAH in the ambient cistern that was missed. The reason may be that when aneurysmal SAH occurred, the bleeding was not only in the ambient cistern but also in the interhemispheric cistern, the cistern of the lateral sulcus, and so forth, which were easy to observe on VNC images.
As described above, the sensitivity and specificity for the diagnosis of $\mathrm{SAH}$ on $\mathrm{VNC}$ at the lesion level were not as good as at the individual level. However, the study also indicated positional and quantitative differences in the "lesions" between TNC and VNC images, and these might be due to the following reasons: First, higher image noise level and more artifacts on VNC images might affect the images and cause a false appearance. Second, in some patients, the level of the section on the VNC images was not exactly the same as that on the TNC images, which resulted in positional differences, especially for the cortical sulci. Finally, the subjective error of observers could also influence the diagnostic results. Nevertheless, the difference at the lesion level between VNC and TNC images did not have a significant effect on the diagnosis of $\mathrm{SAH}$ at the individual level for most patients.

In terms of radiation dose, this study indicated an obvious reduction of volume CT dose index and dose-length product when replacing TNC with VNC images. Compared with subtraction CTA, which is required to provide noncontrast CT as a mask, dual-energy CTA has the advantage that images from 1 single CT acquisition can be used to remove bone structures. This advantage reduced the radiation dose to the patients and eliminated misregistration caused by patient movement during 2 scans. Morhard et $\mathrm{al}^{17}$ and Zhang et $\mathrm{al}^{18}$ compared both the bone removal results of conventional and dual-energy-based techniques and the diagnostic value for intracranial aneurysms and ultimately concluded that dual-energy-based bone removal led to a significant reduction of radiation dose and that dual-energy CTA had a comparable diagnostic accuracy for the detection of intracranial aneurysms.

There were some limitations to this study. First, SAH was diagnosed solely on the basis of the imaging manifestation without combining the medical history and clinical symptoms, which might affect the diagnostic accuracy. Second, in some cases, the interval of the onset of SAH and CT was too long to show hemorrhage clearly; this interval might affect the sensitivity. Third, the same radiologist reviewed the TNC and VNC images with a 2-month interval, which may have led to bias in the study.

\section{CONCLUSIONS}

Despite the higher image noise level, the VNC images were a reliable tool to diagnose $\mathrm{SAH}$, with the advantage of reducing the radiation dose. However, we should be aware of the possible falsenegative results when the ictus is not acute. Regardless of the limitations, VNC has the potential to become a valid clinical tool in diagnosing patients with possible SAH.

\section{REFERENCES}

1. Papanagiotou P, Roth C, Körner H, et al. Diagnostic imaging of subarachnoid hemorrhage [in German]. Radiologe 2011;51:100-05

2. Biller J, GoderskyJC, Adams HP Jr. Management of aneurysmal subarachnoid hemorrhage. Stroke 1988;19:1300-05

AJNR Am J Neuroradiol 36:855-60 May 2015 www.ajnr.org 
3. Meyers PM, Schumacher HC, Higashida RT, et al, for the American Heart Association. Indications for the performance of intracranial endovascular neurointerventional procedures: a scientific statement from the American Heart Association Council on Cardiovascular Radiology and Intervention, Stroke Council, Council on Cardiovascular Surgery and Anesthesia, Interdisciplinary Council on Peripheral Vascular Disease, and Interdisciplinary Council on Quality of Care and Outcomes Research. Circulation 2009;119: 2235-49

4. Hoh BL, Cheung AC, Rabinov JD, et al. Results of a prospective protocol of computed tomographic angiography in place of catheter angiography as the only diagnostic and pretreatment planning study for cerebral aneurysms by a combined neurovascular team. Neurosurgery 2004;54:1329-40

5. Farzad A, Radin B, Oh JS, et al. Emergency diagnosis of subarachnoid hemorrhage: an evidence-based debate. J Emerg Med 2013; 44:1045-53

6. Achenbach S, Ropers D, Kuettner A, et al. Contrast-enhanced coronary artery visualization by dual-source computed tomography: initial experience. Eur J Radiol 2006;57:331-35

7. Johnson TR, Krauss B, Sedlmair M, et al. Material differentiation by dual energy CT: initial experience. Eur Radiol 2007;17: $1510-17$

8. Ferda J, Novák M, Mírka H, et al. The assessment of intracranial bleeding with virtual unenhanced imaging by means of dual-energy CT angiography. Eur Radiol 2009;19:2518-22

9. Reimann AJ, Davison C, Bjarnason T, et al. Organ-based computed tomographic (CT) radiation dose reduction to the lenses: impact on image quality for CT of the head. J Comput Assist Tomogr 2012; $36: 627$
10. Landis JR, Koch GG. The measurement of observer agreement for categorical data. Biometrics 1977;33:159-74

11. Ko JP, Brandman S, Stember J, et al. Dual-energy computed tomography: concepts, performance, and thoracic applications. J Thorac Imaging 2012;27:7-22

12. Chandarana H, Godoy MC, Vlahos I, et al. Abdominal aorta: evaluation with dual-source dual-energy multidetector $\mathrm{CT}$ after endovascular repair of aneurysms: initial observations. Radiology 2008; 249:692-700

13. Song KD, Kim CK, Park BK, et al. Utility of iodine overlay technique and virtual unenhanced images for the characterization of renal masses by dual-energy CT. AJR Am J Roentgenol 2011;197: W1076-82

14. Mileto A, Mazziotti S, Gaeta M, et al. Pancreatic dual-source dualenergy CT: is it time to discard unenhanced imaging? Clin Radiol 2012;67:334-39

15. Moon JW, Park BK, Kim CK, et al. Evaluation of virtual unenhanced CT obtained from dual-energy CT urography for detecting urinary stones. Br J Radiol 2012;85:e176-81

16. Kim JE, Lee JM, Baek JH, et al. Initial assessment of dual-energy CT in patients with gallstones or bile duct stones: can virtual nonenhanced images replace true nonenhanced images? AJR Am J Roentgenol 2012;198:817-24

17. Morhard D, Fink C, Graser A, et al. Cervical and cranial computed tomographic angiography with automated bone removal: dual energy computed tomography versus standard computed tomography. Invest Radiol 2009;44:293-97

18. Zhang LJ, Wu SY, Poon CS, et al. Automatic bone removal dualenergy CT angiography for the evaluation of intracranial aneurysms. J Comput Assist Tomogr 2010;34:816-24 